\title{
CAPITAL SOCIAL ENTRE EMPRESAS FAMILIARES Y ASOCIACIONES EMPRESARIALES EN CELAYA, GUANAJUATO
}

\section{SOCIAL CAPITAL BETWEEN FAMILY BUSINESSES AND BUSINESS ASSOCIATIONS IN CELAYA, GUANAJUATO}

\section{Ricardo Contreras-Soto1; Alberto Valdés-Cobos2 y Alejandra López-Salazar3}

Profesor-investigador de la División de Ciencias Sociales y Administrativas, Universidad de Guanajuato, campus Celaya-Salvatiera. riconsoto@hotmail.com1. Profesor-investigador de la División de Ciencias Sociales y Administrativas, Universidad de Guanajuato, campus Celaya-Salvatierra. cobos_alberto@hotmail.com2. Profesora-investigadora de la División de Ciencias Sociales y Administrativas, Universidad de Guanajuato, campus Celaya-Salvatierra. alejandra_lopezsalazar@yahoo.com.mx3

\section{RESUMEN}

El presente trabajo es parte de un estudio amplio sobre empresas familiares realizado en Celaya, Guanajuato México (año 2011) con una muestra de 343 entrevistas a dueños o directivos responsables. En este estudio se analiza la relación concreta del capital social que tienen las empresas familiares con las distintas asociaciones empresariales, ya que supuestamente es el fin social de dichas asociaciones. El análisis sebasa en la pregunta abierta: ¿Tiene su empresa comunicación con las asociaciones empresariales (Consejo Coordinador Empresarial, COPARMEX, CANACINTRA...) y cómo percibe esa relación (positiva y/o negativa)? Con ello, se quiere saber qué piensan las micro, pequeñas y medianas empresas familiares $¿ \mathrm{Si}$ existen relaciones con las asociaciones empresariales?, ¿Con quién o quienes tienen dichas relaciones? Y ¿Qué opinan de esas relaciones?. El análisis se basa principalmente en la teoría del capital social de Bourdieu. El estudio es mixto, predominantemente cualitativo y explorativo. Se hace un análisis de los distintos testimonios para cuantificar y representar los factores descriptivos de dichas relaciones.

Recibido: 04 de diciembre de 2011. Aceptado: 02 de marzo de 2012. Publicado como ARTÍCULO CIENTÍFICO en Ra Ximhai 8(3): $365-376$.
Palabras clave: capital social, asociaciones empresariales y empresas familiares.

\section{SUMMARY}

This work is part of a comprehensive study on family businesses held in Celaya, Guanajuato Mexico(year 2011) with a sample of 343 interviews with owners or managers responsible. This study examines the specific relationship capital with family businesses with various business associations, as is supposedly the social purpose of these associations. The analysis is based on the open question: Does your business communication with business associations (CCE, COPARMEX, CANACINTRA ...) and how they perceive the relationship (positive and / or negative)? With it, you want to know what they think micro, small and medium-sized family relationships If there are business associations?, Who or who have such relationships? And do you think of these relationships?. The analysis is based primarily on social capital theory of Bourdieu.

The study is mixed, predominantly qualitative and exploratory. An analysis of the various testimonies to quantify and

\footnotetext{
Agradecemos a nuestros alumnos Jesús Pérez Silva y Alberto Enrique Martínez López por su participación.
} 
represent the descriptive factors of these relationships.

Keywords: social capital, business associations and family businesses.

\section{INTRODUCCIÓN}

Actualmente se ha encontrado la importancia que tiene el capital social en las empresas micro, pequeñas y medianas, sobre todo las familiares: ver a Jiménez y Piña Zambrano (2011) y a Contreras, López y Molina (2011). Este estudio pretende aproximarse a las formas específicas de relación social e impresión que se tiene de las asociaciones empresariales y si realmente se da un beneficio para ambos, como fin de existencia de estas asociaciones, se quiere saber si las empresas familiares de la ciudad de Celaya desconoce la existencia de ellas o si tiene presencia en las empresas familiares. Se supone que las asociaciones empresariales tienen una misión ante sus agremiados, queremos ver ahora ¿Cuál es la percepción de las micro, pequeñas y medianas empresas familiares?

\section{Método}

El método es mixto predominantemente cualitativo, de levantamiento amplio de entrevistas semiestructuradas, parte de la base en un momento describir las categorías que se van descubriendo en la exploración.

\section{Muestra}

De acuerdo a los datos del INEGI (2010) se informa que en el municipio de Celaya

\footnotetext{
Otra de las traducciones sobre capital social y la contribución que hace Putnam sobre las distintas formas de él, definidas de la siguiente manera: A) Bonding: Que vendría a significar algo así como construir lazos hacia adentro: "con los míos". Son vínculos intragrupo y son lazos de exclusividad/exclusión. Los vínculos suelen ser fuertes y se producen en el marco de grupos homogéneos como los amigos y la familia. B) Brindging: Que se refiere al establecimiento de "puentes". Los lazos se establecen con personas de fuera del propio grupo: "con los otros". Relaciones con personas y grupos del "exterior". Se trata de tender puentes hacia
}

existen 1,707 unidades económicas las cuales conforman nuestro universo de análisis. El cálculo de la muestra se hizo con el programa Decision Analyst STATS considerando un margen de error del 5\%; una probabilidad de selección del 50\% y un nivel de confianza del 95\%. Estos parámetros de diseño arrojaron una muestra de 314 empresas, las cuales representan el $18 \%$ del universo. Durante el trabajo de campo se aplicó el cuestionario a 343 empresas familiares establecidas realizando la entrevista a los dueños o responsables de los mismos, con lo cual se obtuvo una representatividad del $20.09 \%$.

De las 343 entrevistas a empresas familiares, las microempresas representan el 72 . $89 \%$ de la muestra; las pequeñas $11.95 \%$ y medianas $0.58 \%$., como lo muestra el siguiente cuadro:

Cuadro 1. Tamaño de las empresas de la muestra. Tamaño de las empresas

Teoría: Capital social

Podemos apoyarnos en la definición de capital social de acuerdo a Gilberto Giménez (citado en Jiménez, 2005: 86):

"Recursos sociales consistentes en la capacidad de movilizar en provecho propio redes de relaciones sociales más o menos extensas, derivadas de la pertenencia a diferentes grupos o "clientelas".

El capital social se va entretejiendo las relaciones de las personas que se circunscriben a un campo social determinado,

amigos, asociados o colegas lejanos. Son lazos de inclusión; que buscan incluir a los otros en "lo propio. C) Linking: Establecer conexiones, vincular. Se refiere a las relaciones con individuos y grupos con diferentes estatus y poder. Hay autores que refieren esta última forma de establecer vínculos a la capacidad de conseguir recursos, ideas e información de instituciones formales más allá de la comunidad. Citado en Capital social y educación s/A Número 185, serie II en la página http://www.apagina.pt/?aba=7\&cat=522 $\&$ doc $=13514 \&$ mid $=2$, (consultado 8/01/2011). 
Cuadro 1. Composición porcentual y nomenclatura de los siete óxidos mixtos.

\begin{tabular}{|l|l|l|}
\hline Oxidos Mixtos & Composición en porcentaje & Nomenclatura \\
\hline $\mathrm{Al}_{2} \mathrm{O}_{3}-\mathrm{ZrO}_{2}$ & $80-20$ & $\mathrm{Al}_{80}-\mathrm{Zr}_{20}$ \\
\hline $\mathrm{Al}_{2} \mathrm{O}_{3}-\mathrm{CeO}_{2}$ & $80-20$ & $\mathrm{Al}_{80}-\mathrm{Ce}_{20}$ \\
\hline $\mathrm{Al}_{2} \mathrm{O}_{3}-\left(\mathrm{Ce}_{0.5}-\mathrm{Zr}_{0.5}\right) \mathrm{O}_{2}$ & $80-10-10$ & $\mathrm{Al}_{80}-\mathrm{Zr}_{10}-\mathrm{Ce}_{10}$ \\
\hline $\mathrm{Al}_{2} \mathrm{O}_{3}$ & 100 & $\mathrm{Al}_{100}$ \\
\hline $\mathrm{CeO}_{2}-\mathrm{ZrO}_{2}$ & $50-50$ & $\mathrm{Ce}_{50}-\mathrm{Zr}_{50}$ \\
\hline $\mathrm{CeO}_{2}-\mathrm{ZrO}_{2}$ & $25-75$ & $\mathrm{Ce}_{25}-\mathrm{Zr}_{75}$ \\
\hline $\mathrm{CeO}_{2}-\mathrm{ZrO}_{2}$ & $75-25$ & $\mathrm{Ce}_{75}-\mathrm{Zr}_{25}$ \\
\hline
\end{tabular}

aunados a múltiples efectos derivados de estos procesos: como la confianza, intereses, la aceptación, etcétera.

\section{Capital social - Linking}

Partimos de la premisa que hay distintos tipos de capital social, entre las distintas formas de capital social es Putnam (2000) citado por García (2010:7) quien nos puede ayudar a ver y entender las relaciones que existe con las cámaras y asociaciones empresariales. De esta manera nos vamos a referir al capital social linking de las empresas familiares y de las asociaciones. Para ello, utilizamos las categorías operativas que encontramos: Sí tiene relación, Coparmex, Canaco, Hacienda, Federación de masa y tortilla del Estado de Guanajuato, Canacintra, Cámara de la Construcción, Sagarpa, SIEM, Unión del Mercado de Morelos. Buenas opiniones y Malas opiniones, no tiene relación con argumento, no tienen relación sin argumento, no saben y no contestó.

\section{Desarrollo}

Si tiene relación

En este apartado se presenta los casos que hablan de que tienen algún tipo de relación con las Cámaras Empresariales.
Se habla que hay un proceso próximo con las cámaras

$\mathrm{Si}$ relación estrecha y comunicación. (Entrevista 25) (Venta de tacos, ---------)

Este testimonio da a entender que sí hay una relación, pero que no se ha establecido mayor provecho en las relaciones.

Gustaría muchas cosas, pero sí. (Entrevista 34) (Manufacturera de metal, 6 trabajadores) Este testimonio nos da a entender que si hay relaciones empresariales con estas asociaciones.

Si (Entrevista 77) (Tortillería, Trabajadores 5, 3 establecimientos)

Este testimonio nos da a entender que si hay relaciones empresariales con estas asociaciones.

Si, bien. (Entrevista 81) (Compra y venta de productos automotrices, 2 trabajadores)

Si tiene relación pero se siente obligado a pertenecer a estas asociaciones.

Si, prácticamente es obligatorio (Entrevista 93) (Fabricación de arpillas)

Este testimonio nos da a entender que si hay relaciones empresariales con estas asociaciones. 
Sí. (Entrevista 151) (Cartón Corrugado, 120 trabajadores)

Este testimonio nos da a entender que si hay relaciones empresariales con estas asociaciones.

Si tienen comunicación. (Entrevista 154) (Súper y Farmacia, 15 trabajadores)

Este testimonio nos dice que si está relacionado, incluso los invitan a conferencias y seminarios a las cuales casi no asiste por falta de tiempo.

$\mathrm{Si}, \mathrm{mmm}$ ! Si la tenemos pero muy poca, también ellos nos invitan también a seminarios que hace el Gobierno del Estado, empresas exitosas, pero por falta de tiempo no asistimos, pero trato de asistir a casi todas. (Entrevista 278) (Panadería, 12 trabajadores)

Este testimonio nos da a entender que si hay relaciones empresariales con estas asociaciones pero no se involucra demasiado.

Si tengo pero no tengo mucho que ver. (Entrevista 163) (Comercial, 3 trabajadores) Este testimonio nos da a entender que si hay relaciones empresariales con estas asociaciones.

Si adquieres mejor asociación o siempre pero si (Entrevista 194) (Industrial comercial, 5 trabajadores)

Coparmex

En este apartado se presenta los casos que hablan de que tienen algún tipo de relación con Coparmex.

Se habla de que si estuvo con esta asociación pero se salió para lograr otros proyectos.

Estuve en la COPARMEX un tiempo pero me salí porque tuve otros proyectos aunque aún tengo comunicación (Entrevista 58) (Postres, 2 trabajadores)

Solo con Coparmex.

Coparmex (Entrevista 97) (-----, 2 trabajadores)

En testimonio nos dice que está con Coparmex, sin embargo no está cien por ciento seguro.

Me imagino que si no recuerdo si es coparmex. (Entrevista 205) (Refaccionaría, 3 trabajadores)

Solo con Coparmex.

COPARMEX (Entrevista 221) (Alimenticio, 5 trabajadores)

Solo con Coparmex.

Estamos con COPARMEX integrados. (Entrevista 245) (Venta de producto de cacahuates con dulce, 10 trabajadores)

En testimonio nos dice que esta con Coparmex, sin embargo no está cien por ciento seguro.

Pues creo que nada más le pagamos a no sé si a CANACINTRA o COPARMEX, no se algún, pero la verdad no sabré decirte. (Entrevista 282) (Comercial, 10 trabajadores)

Solo con Coparmex.

$\mathrm{Si}$ pertenecemos a COPARMEX (Entrevista 322) (Comercial y de servicios, 3 trabajadores)

Solo con Coparmex.

Con Coparmex (Entrevista 323) (Servicios, 8 trabajadores)

Canaco

En este apartado se presenta los casos que hablan de que tienen algún tipo de relación con Canaco.

Solo con Canaco. 
Canaco (Entrevista 2) (Abarrotes, 17 trabajadores)

Solo con Canaco, y le agrada que sean directos con ellos.

Pues solamente con CONACO y la relación es muy directa, o sea solo a lo que vas. (Entrevista 19) (Compra-venta de ropa, 2 trabajadores)

Estas asociaciones les llevan el papeleo y los permisos de todo.

Si con CANACO Y CANACINTRA, estas nos llevan todo el papeleo permisos y todo. Entrevista 172) (Fabrica de cajetas y dulces, 5 trabajadores)

Este testimonio nos dice que esta solo por obligación, ya que así se lo exige la Cámara de Comercio.

Si nos obligan con la cámara de comercio nos tenemos que afiliar a CANACO. (Entrevista 203) (Refacciones automotrices nuevas, no especifica trabajadores)

Solo con Canaco.

Canaco (Entrevista 304) (--------------)

No tiene demasiada relación con esta asociación sin embargo dice que cada año se aparece a cobrar su cuota.

CANACINTRA Y CANACO, no tanto en la relación pero no fallan cada año en cobrar su cuotas (Entrevista 305) (------------------) Solo con Canaco.

A LA CAMARA DE COMERCIO, CANACO SERVITUR (Entrevista 309) (Comercio, 4 trabajadores)

Solo con la Cámara de Comercio.

Con la cámara de Comercio (Entrevista 59) (Carnes, 6 trabajadores)

Esta con la Cámara de Comercio hay buena relación porque esta asociación me asesora de manera correcta.

Si el comercio pues está muy bien porque nos apoyan nos asesoran (Entrevista 75) (Tortillería, 8 trabajadores)

Solo está con la Cámara de Comercio.

No, nada más con la Cámara de Comercio (Entrevista 152) (Comercial, 2 trabajadores) Solo con la Cámara de Comercio pero no hay buena relación debido a que está afiliado a otra asociación.

Con la de comerciantes aunque no de manera directa ya que estamos en una afiliación. (Entrevista 171) (Comercio, 3 trabajadores) Solo esta con la Cámara de Comercio.

Con la cámara de comercio solamente. (Entrevista 201) (Comercio, 5 trabajadores) Solo está con la Cámara de Comercio.

A la cámara de comercio, CANACO SERVITUR (Entrevista 309) (Comercio, 4 trabajadores)

Tiene relación con la Cámara de Comercio y con la Unión de Hot Dog y Hamburguesas único en Celaya.

Pues solo con la cámara de comercio y con la unión de hot dog y hamburguesas único en Celaya. (Entrevista 311) (Comercio, 6 trabajadores)

Está relacionada con varias asociaciones.

CON LA ASOCIACION MEXICANA DE COMERCIO, CON HACIENDA CON PRECIDENCIA, IMEC, FISCALIZACION, ETC... (Entrevista 312) (Servicio, 4 trabajadores)

Solo con la Cámara de Comercio. LACAMARADEPEQUEÑOCOMERCIO. (Entrevista 313) (Comercio y servicio, 4 
trabajadores)

Tiene relación con la Cámara de Comercio y con la Unión de Hot Dog y Hamburguesas único en Celaya.

SI CON LA UNION DE HOT DOGS Y HAMBURGUESAS Y CON LA DE PEQUEÑO COMERCIO. (Entrevista 314) (Servicios, 3 trabajadores)

Hacienda

En este apartado se presenta los casos que hablan de que tienen algún tipo de relación con Hacienda.

Este testimonio nos da a entender que la persona no entendió la pregunta ya que Hacienda no es una asociación empresarial.

No, nada más con Hacienda. (Entrevista 89) (Aguas frescas y refrescos, 2 trabajadores) Este testimonio nos da a entender que la persona no entendió la pregunta ya que Hacienda no es una asociación empresarial.

No, solo con Hacienda. (Entrevista 174) (Venta de pollo y huevo fresco, 3 trabajadores)

Federación de masa y tortilla del Estado de Guanajuato

En este apartado se presenta los casos que hablan de que tienen algún tipo de relación con la Federación de masa y tortilla del Estado de Guanajuato.

Solo con la Federación de masa y tortilla del estado de Guanajuato.

Federación de masa y tortilla del estado de Gto. (Entrevista 63) (Tortillería, 3 trabajadores)

Canacintra

En este apartado se presenta los casos que hablan de que tienen algún tipo de relación con Canacintra.

Este testimonio está satisfecho con Canacintra porque le ha ayudado en obtener permisos.

Si con CANACINTRA, bien o sea nos ha ayudado en dos que tres situaciones más que nada en permisos y todo eso. (Entrevista 90) (Elaboración y venta de galletas y pasteles, 6 trabajadores)

Si solo tienen relación con Canacintra.

Tiene relación con la CANACINTRA. (Entrevista 107) (Sastrería, 1 trabajador)

Si solo tienen relación con Canacintra.

Con canacintra. Estamos afiliados. (Entrevista 129) (Venta de ropa, 4 trabajadores)

Estas asociaciones les llevan el papeleo y los permisos de todo.

Si con CANACO Y CANACINTRA, estas nos llevan todo el papeleo permisos y todo. Entrevista 172) (Fabrica de cajetas y dulces, 5 trabajadores)

Estaba con Canacintra y ahora está con un contador público.

Estaba con CANACINTRA antes, a hora estoy con un contador público. (Entrevista 192) (Venta de medicamentos, 5 trabajadores)

Tiene relación con alguna asociación pero no sabe cuál es.

Pues creo que nada más le pagamos a no sé si a CANACINTRA o COPARMEX, no se algún, pero la verdad no sabré decirte. (Entrevista 282) (Comercial, 10 trabajadores)

Solo con Canacintra. 
CANACINTRA No. ayudas de esas No. (Entrevista 289) (Panadería, 3 trabajadores) No tiene estrecha comunicación, sin embargo cada año se presentan a cobrar su cuota.

CANACINTRA Y CANACO, no tanto en la relación pero no fallan cada año en cobrar su cuotas (Entrevista 305)

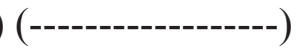

\section{Cámara de Construcción}

En este apartado se presenta los casos que hablan de que tienen algún tipo de relación con Cámara de Construcción.

Solo con la Cámara de Construcción.

Si, con la cámara de construcción. (Entrevista 198) (Diseño y construcción, 2 trabajadores)

\section{Sagarpa}

En este apartado se presenta los casos que hablan de que tienen algún tipo de relación con Sagarpa.

\section{Solo con Sagarpa.}

Si con SAGARPA por los animales que están ahí anotados. (Entrevista 235) (Comercio, no especifica trabajadores)

Nos apoyan y asesoran para mejorar como empresa.

SAGARPA, Ingenieros agrónomos parasitólogos (IAP), Comité Estatal de Sanidad del Estado de Guanajuato, AMIFAC (Asociación Mexicana de Insumos y Fertilizantes AC). Nos brindan apoyo y asesoría lo cual nos ayuda para mejorar. (Entrevista 259) (Compra-Venta de productos agrícolas, 21 trabajadores)

\section{SIEM}

En este apartado se presenta los casos que hablan de que tienen algún tipo de relación con SIEM.

Solo esta con la SIEM sin embargo no está satisfecha porque solo cobran y no le asesoran de manera adecuada.

Ahorita solamente tengo con el "SIEM" que es el sistema de información empresarial mexicano, pero la verdad como que no explican mucho solo vienen a cobrar la cuota y si solo una va y pregunta hay medio le informan. (Entrevista 284) (Productos de Limpieza, 1 trabajador)

Unión del Mercado de Morelos

En este apartado se presenta los casos que hablan de que tienen algún tipo de relación con La Unión de Mercado de Morelos.

Solo con la Unión de Mercado de Morelos. UNION DEL MERCADO MORELOS (Entrevista 310) (Comercio, no específica trabajadores)

Percepciones u opiniones positivas

En este apartado se presenta los casos que hablan de buenas opiniones en relación con estas asociaciones empresariales.

Este testimonio nos habla de ser emprendedores para poder ayudar a la sociedad.

Que sigamos emprendiendo negocios ya que así ayudamos a nuestra sociedad. (Entrevista 12) (------- 5 trabajadores)

A esta empresa le gustaría que la Asociación lo diera a conocer en las universidades públicas y privadas.

Pues en que nos dé más a conocer con las demás universidades. (Entrevista 17) (Fabricación de Válvulas y bebederos para ganado, 12 trabajadores)

Esta asociación debe establecer precios 
adecuados en todas las empresas de este giro.

Que establezcan precios adecuados para la sociedad (Entrevista 22) (Lácteos y carnes frías, 3 trabajadores)

Las empresas necesitan un producto de calidad.

Que ofrezcan producto de calidad(Entrevista 86) (Hielitos, 15 trabajadores)

En este testimonio nos damos cuenta que el entrevistado no sabía que decir.

Este que le echen ganas. (Entrevista 88) (Bisutería, 4 trabajadores)

Con este testimonio podemos darnos cuenta que a los empresarios no les va ni mejor ni peor al estar involucrado con alguna asociación.

Lo tuve un tiempo, pero me retire un poco, son buenas pero es como todo cuando estaba con ellos no me iba tan mal pero tampoco tan bien, las asociaciones es para cierto circulo... (Entrevista 96) (Productos de limpieza, 10 trabajadores)

Este empresario no está relacionado con ninguna asociación pero piensa que si lo estuviera podría crecer más como empresa.

No. Eso también ayudaría mucho a la empresa para poder crecer más. (Entrevista 130) (4 trabajadores)

Este empresario está contento con el trabajo que hace esta asociación.

$\mathrm{Si}$, fíjate que es muy beneficiosa, ya que en muchos de los casos te orientan en cuanto a los problemas que se presenten. (Entrevista 212) (Tortillería, 7 trabajadores)

En este testimonio nos damos cuenta que este empresario no sabía que decir.

Que le echemos ganas para salir adelante
(Entrevista 252) (Control de calidad para materiales de construcción, 4 trabajadores) Hay alguna relación pero no vemos que nos apoyen en nada.

Si hay relación pero básicamente no nos han apoyado en nada. (Entrevista 253) (Venta de novias y accesorios, 10 trabajadores) Existe relación y nos brindan información a través de folletos, cuestionarios o volantes escasamente una vez al año.

En algunas ocasiones si porque nos mandan folletos, nos mandan ah volantes, nos mandan algunas cuestiones importantes que pueden hacer, pero mucho contacto no, probablemente, sería una vez al año. (Entrevista 292) (Comercial, 3 trabajadores) Este empresario dice que con actitud todo se puede.

Que le echen ganas a su negocio que todo se puede lograr y más con ganas (Entrevista 330) (Comercio, 4 trabajadores)

Este empresario dice que el sol sale para todos.

Pues que para todos hay (entrevista 333) (Muebles Rústicos, no especifica trabajadores)

Este empresario dice que el que persevera alcanza.

Que sean perseverantes, como dice el dicho, "El que persevera, alcanza". (Entrevista 339) (Comercial, 7 trabajadores)

Percepciones u opiniones negativas

En este apartado se presenta los casos que hablan de que tienen malas opiniones con estas asociaciones.

No tiene relación con ninguna asociación pues sólo cobran y no obtienen beneficio alguno. 
No a ninguna solo te cobran y no vez beneficio. (Entrevista 105) (Textil, 3 trabajadores)

A este empresario lo han invitado a pertenecer a alguna asociación pero él no ve ningún beneficio.

No, si me han invitado pero no he querido entrar, parte de que te cobran no me beneficia en nada. (Entrevista 106) (Restaurantero, de 10 a 30 personas)

Este empresario nos dice que cobran su cuota pero no te ayudan en nada.

Nada más pagamos todos los cobros que llegan y ni conocemos y sabemos en qué nos ayudan (Entrevista 108) (Carnicería, 6 trabajadores)

A través de este testimonio nos damos cuenta que este empresario si ha pertenecido a varias asociaciones pero solo es una pérdida de tiempo y dinero.

No, porque para mí lo que me a servido Coparmex, Canacintra y todo ese tipo de empresas para cobrarme el tiempo, porque no me a apoyado en nada. Mala porque nunca se acercan a nosotros más que solo a cobrarnos el tiempo. (Entrevista 128) (Venta de ropa, 4 trabajadores)

Estos comerciantes pagan impuestos pero son inconformes.

Pues nosotros estamos en la cámara de pequeños comerciantes y pues no nos apoyan en nada, nada más es pagar el impuesto ese. (Entrevista 132) (5 trabajadores)

Este comerciante dice que lo roban.

Ninguna. Mala solo roban. (Entrevista 160) (Venta de herramienta, 5 trabajadores)

A este comerciante solo le cobran y no le ayudan en nada.

Ninguna. Yo la veo mala porque solo te cobran. (Entrevista 190) (Venta de dulces al mayoreo, 3 trabajadores)

Este comerciante ve estancado el crecimiento de su empresa cuando está relacionado con estas asociaciones.

Antes se tenía una relación con la asociación del mercado de abastos, pero esta relación en vez de beneficiar el crecimiento de mi empresa, se vio entorpecido. (Entrevista 255) (Venta de Desechables, 3 trabajadores) A este comerciante solo lo roban estas asociaciones.

Ninguna. Mala solo roban. (Entrevista 317) (Venta de Herramientas, 5 trabajadores).

Este comerciante tiene una idea de interés político sobre estas asociaciones.

Mmm... no porque por lo que se esas asociaciones están muy apegadas a lo que son campañas políticas y por eso como que no. (Entrevista 279) (De servicios, 3 trabajadores)

No le interesa tener relación porque no hay beneficio.

No porque no es mucho el beneficio (Entrevista 319) (Comercial, 8 trabajadores) No le interesa porque dice que no tiene provecho.

Actualmente no estábamos por entrar a Coparmex porque se me hizo una pérdida de tiempo estar asistiendo a sus reuniones y realmente el provecho que le sacaba uno era muy mínimo. (Entrevista 326) (Comercial y de servicios, 35 trabajadores)

A este comerciante le cobran cuota de empresa grande.

Tuve un tiempo, pero ahora ya no, porque había que cooperar y yo ya no quise cooperar, si lo que pasa es que cuando nosotros 
hemos estado, este, ahí son empresas que ponen mucho dinero, $\mathrm{y}$ entonces a la hora de las cuotas nos ponen al parejo con esas empresas, y pus no, no alcanzamos a dar la misma cooperación que esas empresas (Entrevista 335) (Artesanías de Madera, no especifica trabajadores)

No, con argumento

En este apartado se presenta los casos que hablan de que no tienen algún tipo de relación con alguna Cámara empresarial.

Esta persona dice que no le interesa tener relación con ella.

No tengo relación con las cámaras de la industria y comercio y por el momento no me interesa. (Entrevista 8) (Producto naturista, 2 trabajadores)

No existe relación sin embargo nos invitan a eventos que tienen.

No tenemos relación directa, sin embargo si nos invitan a desayunos, conferencias, etc. (Entrevista 39) (Maquila de fertilizantes agrícolas y urbanos, 33 trabajadores)

No tiene relación porque no tiene mucho la empresa.

No con ninguna asociación y mi argumento es por el poco tiempo que tiene la empresa. (Entrevista 57) (Comercio,

No tiene ninguna relación con nadie.

No tiene relación con este tipo de asociaciones. (Entrevista 71) (Tortillería, 2 trabajadores)

No tiene alguna relación con alguien.

No, no existe ninguna comunicación con las anteriores. ((Entrevista 72) (Comercial, 5 trabajadores)

A este comerciante va pensando en el futuro.
No, pero estamos en proceso (Entrevista 114) (Abarrotes, 3 trabajadores)

No tiene alguna relación con alguien.

No, no la tenemos trabajamos independiente. (Entrevista 123) (Creación de bolsas y estampados, 6 trabajadores)

Este comerciante confía en sus habilidades.

No, yo mismo busco la manera de promoverme. (Entrevista 153) (Industrial, 4 trabajadores)

Este comerciante esta encasillado en una idea.

No. Nada más así como los directivos de aquí de la unión son lo que llevan eso. (Entrevista 185) (Venta de agua y nieve, 8 trabajadores)

Este empresario piensa que una pequeña empresa no necesita asesoramiento.

No, si eeh! escuchado de ellas y de hecho en alguna ocasión he ido a algún congreso o algunas platicas, pero realmente como es...., volvemos a lo mismo, como es una pequeña empresa, no eh requerido sus asesoramiento de ellos. (Entrevista 243) (--------, 1 trabajador)

Este comerciante aún no tiene relación pero no descarta la posibilidad.

No tengo ninguna relación con ellos, pero si me gustaría para poder agrandar mi negocio, ver la manera. (Entrevista 249) (Poner uñas, 3 trabajadores)

No hay relación porque no los han buscado ni la asociación a ellos.

No, no tenemos ninguna relación hasta ahorita, ni hemos recibido invitación, ni se nos han acercado. (Entrevista 261) (Constructora, 30 personas)

No hay relación pero no descarta la posibilidad. 
No, no hasta ahorita todavía no, tal vez más a futuro en cuanto a poder pues solicitar apoyo eh... no se para de gente preparada eh... para poderla contratar. (Entrevista 280) (Comercial, 4 trabajadores)

Este comerciante confía en sus habilidades. No. Estamos luchando poco a poquito y vamos paso a pasito. (Entrevista 327) (Textil, 8 trabajadores)

No saben

En este apartado se presenta los casos que hablan de que no saben.

Pues la verdad de eso si no tengo idea, estamos con hacienda pero no sé. (Entrevista 276) (Peletería, 3 trabajadores)

Mi marido tenía ¿sabe con cuál? Ahorita no se te decir. (Entrevista 275) (Producción de pan, 4 trabajadores)

No sabría responderte de esa pregunta pero debe haber comunicación con otras empresas (Entrevista 306) (------------)

¿Si hay sindicato de carniceros? (Entrevista 307) (---------)

Sabrá Dios que sea eso (Entrevista 112) (4 trabajadores)

No solo mi hermano sabe. (Entrevista 226)

\begin{tabular}{|c|c|c|c|c|c|}
\hline \multirow[b]{2}{*}{$\begin{array}{l}\text { Tiene relación con } \\
\text { Asociaciones } \\
\text { Empresariales }\end{array}$} & \multicolumn{4}{|c|}{ Tamaño de la empresa } & \multirow[b]{2}{*}{ Total } \\
\hline & Micro & Pequeña & Mediana & $\mathrm{Nc}$ & \\
\hline Si tiene relación & 4 & 3 & 1 & 4 & 12 \\
\hline $\begin{array}{l}\text { Sï; Unión del mercado de } \\
\text { Morelos }\end{array}$ & 0 & 0 & 0 & 1 & 1 \\
\hline Sí, COPARMEX & 5 & 1 & 0 & 1 & 7 \\
\hline Si, CANACO & 2 & 1 & 0 & 1 & 4 \\
\hline $\begin{array}{l}\text { SÏ, Federación de masa y } \\
\text { tortilla del Estado de } \\
\text { Guanajuato }\end{array}$ & 10 & 0 & 0 & 0 & 10 \\
\hline SÍ, CANACINTRA & 1 & 0 & 0 & 0 & 1 \\
\hline $\begin{array}{l}\text { SÏ, CAMARA DE LA } \\
\text { CONSTRUCCIÓN }\end{array}$ & 1 & 0 & 0 & 0 & 1 \\
\hline SÏ, SAGARPA & 6 & 1 & 0 & 1 & 8 \\
\hline Sí, HACIENDA & 1 & 0 & 0 & 0 & 1 \\
\hline Si, SIEM & 1 & 1 & 0 & 1 & 3 \\
\hline Buenas opiniones & 9 & 3 & 0 & 2 & 14 \\
\hline Malas opiniones & 9 & 2 & 0 & 2 & 13 \\
\hline No con Argumento & 10 & 1 & 0 & 3 & 14 \\
\hline No saben & 2 & 0 & 0 & 2 & 4 \\
\hline No tienen relación & 166 & 23 & 1 & 31 & 221 \\
\hline $\mathrm{Nc}$ & 23 & 5 & 0 & 1 & 29 \\
\hline Total & 250 & 41 & 2 & 50 & 343 \\
\hline
\end{tabular}

(Tortillería, 4 trabajadores)

No tiene relación (sin argumento)

En este apartado se indican los casos que hablan de que no tienen algún tipo de relación con alguna cámara o asociación empresarial, se manifiestan 221 casos, de los cuales 166 son micro empresas, 23 son pequeñas, 1 es mediana y 31 no se sabe de qué tamaño son.

A manera de conclusión

De acuerdo a los resultados encontramos que las empresas familiares:

1. El $14 \%$ aproximadamente de las empresas si tienen relación con las cámaras y asociaciones.

2. Mientras que no tiene relación con cámaras y asociaciones es el 68.51\%

3. Solo el $1.16 \%$ no sabe sobre las cámaras y asociaciones.

4. De lo que se desprende es que las cámaras y asociaciones trabajan con un porcentaje muy pequeño de empresas familiares, es poco el capital social que se ha establecido con ellos.

5. Entre buenas opiniones en general sol el $4.08 \%$ son motivacionales, otros agradecen la información que proporcionan.

6. Las malas opiniones son el $3.79 \%$ muchas de las opiniones dicen que no ven la diferencia existente en beneficios con las asociaciones y las cámaras, que son caras, que nos son equitativas, que se dedican a la política, sienten que cobran caro, algunos empresarios prefieren no involucrarse con ellos debido a los costos. Los empresarios sienten que no les ayudan en nada.

7. Ni Hacienda, ni el SIEM es una asociación empresarial, aunque el SIEM lo maneja o promueve alguna de las asociaciones.

8. De las cámaras se apoyan en la Federación de Masa y Tortilla del Estado el 

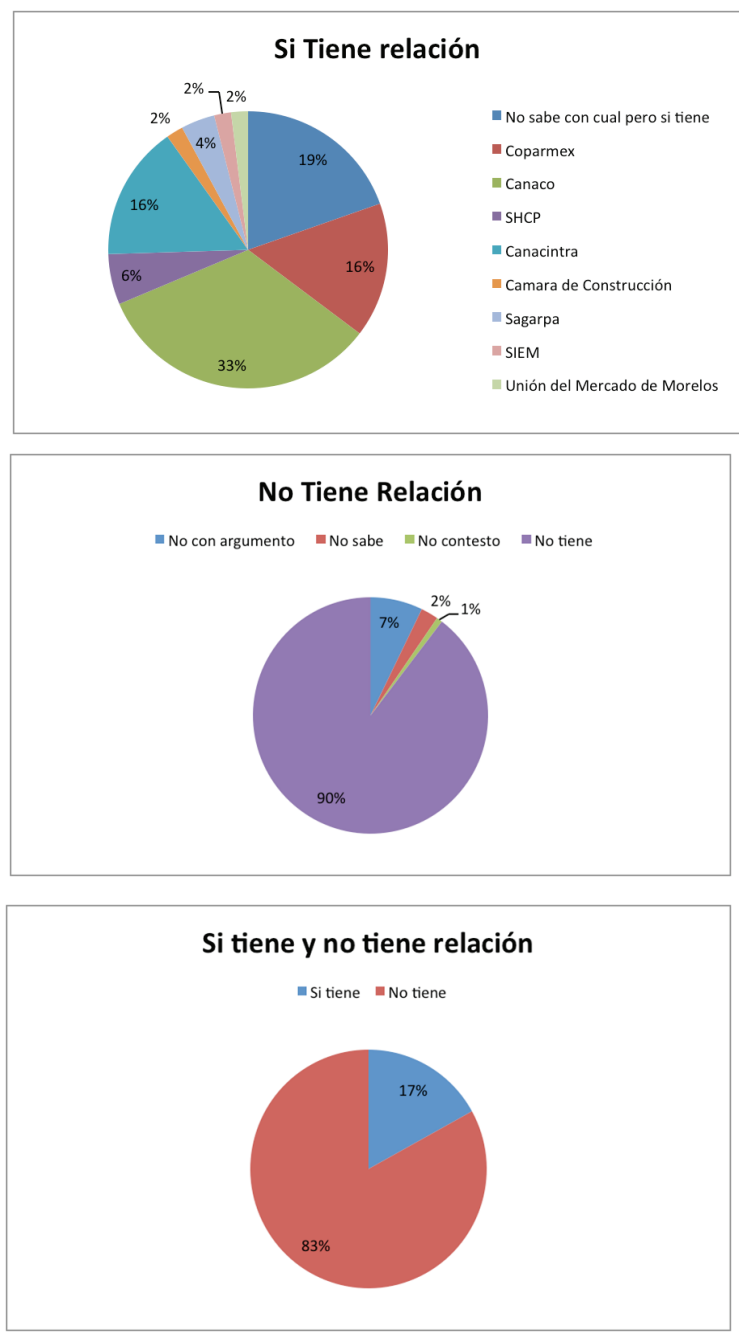

$2.49 \%$ principalmente micros.

9. Las cámaras y asociaciones empresariales tiene que trabajar estratégicamente más y ofertar más servicios a las empresas familiares de acuerdo a sus necesidades reales.

10. Se deben construir estrategias de acuerdo a las exigencias de las empresas familiares ohacerasociaciones empresariales ideales a estos casos mayoritarios.

\section{LITERATURA CITADA}

Bourdieu, P. 1990. Sociología Y Cultura. México. Editorial Grijalbo.

Contreras R., López A. Y Ríos M. 2011.
Cultura Empresarial: Tipología Social De Los Valores En La Micro, Pequeña Y Mediana Empresa En Celaya. Ocma. Universidad De Guanajuato.

Contreras R. Y Regalado R. 2008 a Potencialidades Y Problemas En Empresas De Participación Familiar En Las Mipymes. Edición Electrónica Gratuita. Texto Completo En Www. Eumed.Net/Libros/2008C/437/ Isbn-13: 978-84-691-7211-7.

Contreras R., López A. Y Molina R. 2011. Emprendimiento: Dimensiones Sociales Y Culturales En Las Mipymes. México. Pearson.

Contreras R., López A. Y Ríos M. 2011. Cultura Empresarial. Tipología Social De Los Valores En La Mipyme. México. Ocma.

García, L. 2005. "Estudios De Empresas Familiares En Escenarios Partiendo Del Modelo Evolutivo Tridimensional". Revista Omnia. Maracaibo Venezuela. Universidad De Zulia. Pp: 29-52. Consultada En La Red De Revistas Científicas De La America Latina Y El Caribe, España Y Portugal.

González M., Guzmán A., Pombo C. Y Trujillo M. 2010. Empresas Familiares: Revisión De La Literatura Desde Una Perspectiva De La Agencia. Cuadernos De Administración, Vol. 23, Núm. 40, Enero- Junio 2010, Pp. 11-33 Pontífica Universidad Javeriana. Colombia.

Jiménez I. 2005. Ensayos Sobre Pierre Bourdieu Y Su Obra. México. Coedición Universidad Autónoma De México Unam Y Plaza Y Valdez. Jiménez, M. Y Piña Zambrano, H. 2011. 
"Capital Social En Empresas Familiares". Revista Venezolana De Gerencia, Vol. 16, Núm. 54, Abril Junio 2011, Pp. 255-273 Universidad Del Zulia Maracaibo Venezuela.

Payne, M. (Comp.) 2002. Diccionario De Teoría Crítica Y Estudios Culturales. Buenos Aires, Argentina. Paidós.

Putnam, R. 2000. Bowling Alone: The Collapse And The Revival Of American Community. Simon And Schuster. New York.

Rabell, C. (Coordinadora). 2009. Tramas Familiares En México Contemporáneo. Una Perspectiva Sociodemográfica. México. Coedición Instituto De Investigaciones Sociales Unam Y El Colegio De México.

Ricardo Contreras Soto, Licenciado en Administración por la Universidad Nacional Autónoma de México; Maestro en Antropología por la Universidad Autónoma de Querétaro; Doctorado en Administración por la Universidad de Celaya. Actualmente es SNI nivel 1. Hizo el diplomado de investigación cualitativa Cultura y Comunicación en la Universidad Iberoamericana de León. Terminó el Diplomado de la Cuestión Étnica ENAH - UAQ. Participó de manera satisfactoria en el Diplomado de Cluster del Instituto Tecnológico de Monterrey en Michoacán. Trabaja en la Universidad de Guanajuato Campus Celaya - Salvatierra $\mathrm{y}$ en la Universidad de Celaya; publicaciones: Emprendimiento: dimensiones sociales y culturales en las Mipymes de Ricardo Contreras Soto, Alejandra López Salazar y Rubén Molina Sánchez, editado por PEARSON; Contreras Soto,
R.: (2007). Es miembro del Cuerpo Académico en Formación: Gestión y Desarrollo de Micros, Pequeñas y Medianas Empresas (MIPYMES). Correo electrónico: riconsoto@ hotmail.com.

Alberto Valdés Cobos, Doctor en Ciencias Agrarias por la Universidad Autonoma Chapingo (UACh). Realizo sus estudios de licenciatura y maestría en Sociología Rural en la UACh. Ha impartido los cursos de Sociología General, Sociología de las Organizaciones, Sociología Rural, Desarrollo Sustentable y Prospectiva, entre otros, en la Universidad del Valle de México (UVM), campus Texcoco; la Universidad Autonoma Chapingo; el Centro Superior de Estudios Tecnológicos de Teposcolula (CESETT), Oaxaca; y la Universidad Autonoma Metropolitana (UAM), unidad Azcapotzalco. Ha publicado una diversidad de artículos de temática sociológica. La Universidad de Guanajuato público su libro Hacia la sociología de los futuros. Una aproximación al Homo futurus complexus. Actualmente es profesor-investigador de tiempo completo de la División de Ciencias Sociales y Administrativas de la Universidad de Guanajuato, campus Celaya-Salvatierra. Es miembro del Cuerpo Academico en Formación: Género y Políticas Públicas para el Desarrollo Social y Humano. Correo electronico: cobos_alberto@ hotmail.com

Alejandra López Salazar, Doctora en Negocios y Estudios Económicos por la Universidad de Guadalajara. Profesora-Investigadora de la Universidad de Guanajuato Campus 
Celaya-Salvatierra. Actualmente es SNI nivel 1. Líneas de investigación: Competitividad y Colaboración; Responsabilidad Social y Sustentabilidad. Dictaminadora de trabajos de investigación de Revistas nacionales e internacionales. Miembro de la Comisión de Titulación del Programa Educativo de la Licenciatura en Administración y de la Mestría en Fiscal. Miembro de la Comisión Evaluadora de la División de Ciencias Sociales y Administrativas. Miembro del Consejo Divisional. Evaluadora de ponencias de Congresos nacionales e internacionales. Organización de eventos académicos nacionales e internacionales. Coordinadora de mesa en distintos congresos nacionales e internacionales. Miembro del Cuerpo Académico en Formación: Gestión y Desarrollo de Micros, Pequeñas y Medianas Empresas (MIPYMES). Correo electrónico: alejandra lopezsalazar@yahoo.com.mx 